Supporting Information

\title{
Evaluation of the history of safe use of the maize ZMM28 protein
}

Jennifer A. Anderson, Sarah Brustkern, Bin Cong, Lora Deege, Bryan Delaney, Bonnie Hong,

Shai Lawit, Carey Mathesius, Jean Schmidt, Jingrui Wu, John Zhang, Cindi Zimmermann

Corteva Agriscience ${ }^{\mathrm{TM}}$, Agriculture Division of DowDuPont, Johnston, IA 50131, USA

*Corresponding Author

Jennifer Anderson

Corteva Agriscience ${ }^{\mathrm{TM}}$

Agriculture Division of DowDuPont

Johnston, IA 50131, USA

$515-535-3730$

Jennifer.anderson@corteva.com 
Supporting Information

Table S1: Primer pairs used in RT-PCR.

\begin{tabular}{|l|l|l|l|}
\hline Upstream & Sequence & Downstream & Sequence \\
Primer Name & & Primer Name & \\
\hline DP202216 5'-1 & caaatccaccegtcggcacctccgcttc & DP202216 3'-1 & gatgcccacattatagtgattagcatgtcactatgtgtg \\
\hline$z m m 28-5 '-1$ & cgtggtttcgccaaaccgcttc & $z m m 28-3^{\prime}-1$ & gtcaagacacttgaatactgaaacgtacag \\
\hline$z m m 28-5 '-2$ & ctcttctcctagctctcttgcctg & $z m m 28-3 '-3$ & cacaggacagaggaaacatagtagtg \\
\hline
\end{tabular}


Supporting Information

Table S2 - Site Locations

\begin{tabular}{|c|c|c|}
\hline Site Location & Soil Type & 3-year Cropping History \\
\hline \hline Johnston, Iowa, USA & Loam/Clay Loam & Soybean (2014), Maize (2015), Soybean (2016) \\
\hline Sheridan, Indiana, USA & Loam & Soybean (2014), Maize (2015), Soybean (2016) \\
\hline Fisk, Missouri, USA & Loam & Rice (2014), Soybean (2015), Maize (2016) \\
\hline York, Nebraska, USA & Silt Loam & Soybean (2014), Maize (2015 and 2016) \\
\hline Guelph, Ontario, Canada & Loam & Maize (2014), Soybean (2015), Wheat (2016) \\
\hline Germansville, Pennsylvania, USA & Clay Loam & Wheat (2014), Alfalfa/Orchard grass (2015 and 2016) \\
\hline
\end{tabular}


Table S3. Monthly Temperature, Rainfall, and Irrigation Data

\begin{tabular}{|c|c|c|c|c|c|c|c|c|c|c|}
\hline \multirow{3}{*}{ Site } & \multirow{3}{*}{ Month } & \multicolumn{5}{|c|}{2017 Weather Data } & \multicolumn{4}{|c|}{ Historical Weather Data } \\
\hline & & \multicolumn{3}{|c|}{ Temperature $\left({ }^{\circ} \mathrm{F}\right)$} & \multirow{2}{*}{$\begin{array}{c}\text { Total } \\
\text { Rainfall } \\
\text { (inches) }\end{array}$} & \multirow{2}{*}{$\begin{array}{c}\text { Total } \\
\text { Irrigation } \\
\text { (inches) }\end{array}$} & \multicolumn{3}{|c|}{ Temperature $\left({ }^{\circ} \mathrm{F}\right)$} & \multirow{2}{*}{$\begin{array}{c}\text { Average } \\
\text { Total } \\
\text { Rainfall } \\
\text { (inches) }\end{array}$} \\
\hline & & $\operatorname{Max}$ & Min & Mean & & & Max & Min & Mean & \\
\hline \multirow{6}{*}{$\begin{array}{l}\text { Johnston, } \\
\text { Iowa }\end{array}$} & May & 71 & 50 & 61 & 2.1 & $\overline{0}$ & 72 & 51 & 62 & 4.5 \\
\hline & June & 86 & 60 & 73 & 4.1 & 0 & 81 & 61 & 71 & 5.3 \\
\hline & July & 90 & 65 & 78 & 2.6 & 0 & 85 & 65 & 75 & 4.8 \\
\hline & August & 83 & 57 & 70 & 3.4 & 0 & 83 & 63 & 73 & 4.0 \\
\hline & September & 84 & 56 & 70 & 5.0 & 0 & 76 & 54 & 65 & 3.2 \\
\hline & October & 71 & 52 & 62 & 3.9 & 0 & 63 & 42 & 53 & 2.5 \\
\hline \multirow{7}{*}{$\begin{array}{l}\text { Sheridan, } \\
\text { Indiana }\end{array}$} & May & 76 & 56 & 66 & 0.010 & 0 & 72 & 51 & 62 & 4.1 \\
\hline & June & 82 & 61 & 72 & 5.3 & 0.50 & 80 & 61 & 71 & 5.0 \\
\hline & July & 84 & 65 & 75 & 8.9 & 0 & 83 & 63 & 73 & 4.5 \\
\hline & August & 81 & 59 & 70 & 0.76 & 2.0 & 82 & 62 & 72 & 3.4 \\
\hline & September & 79 & 55 & 67 & 0.77 & 0 & 76 & 54 & 65 & 3.1 \\
\hline & October & 67 & 48 & 58 & 4.3 & 0 & 64 & 43 & 54 & 3.0 \\
\hline & November & 59 & 43 & 51 & 2.5 & 0 & 50 & 34 & 42 & 3.5 \\
\hline \multirow{5}{*}{$\begin{array}{l}\text { Fisk, } \\
\text { Missouri }\end{array}$} & May & 83 & 62 & 73 & 2.4 & 0.60 & 79 & 58 & 69 & 7.5 \\
\hline & June & 88 & 67 & 78 & 2.4 & 2.0 & 89 & 69 & 79 & 3.0 \\
\hline & July & 92 & 72 & 82 & 5.4 & 2.1 & 90 & 70 & 80 & 4.7 \\
\hline & August & 88 & 67 & 78 & 2.0 & 3.1 & 90 & 69 & 80 & 4.1 \\
\hline & September & 82 & 58 & 70 & 0.070 & 0 & 83 & 61 & 72 & 3.4 \\
\hline \multirow{6}{*}{$\begin{array}{c}\text { York, } \\
\text { Nebraska }\end{array}$} & May & 73 & 51 & 62 & 7.1 & 0 & 74 & 51 & 63 & 4.9 \\
\hline & June & 88 & 62 & 75 & 5.1 & 0.60 & 84 & 61 & 73 & 3.8 \\
\hline & July & 89 & 66 & 78 & 6.2 & 1.8 & 88 & 65 & 77 & 3.4 \\
\hline & August & 80 & 59 & 70 & 5.9 & 2.1 & 85 & 63 & 74 & 3.3 \\
\hline & September & 80 & 56 & 68 & 3.9 & 0 & 78 & 53 & 66 & 2.4 \\
\hline & October & 69 & 45 & 57 & 4.5 & 0 & 66 & 40 & 53 & 1.9 \\
\hline \multirow{7}{*}{$\begin{array}{l}\text { Guelph, } \\
\text { Ontario }\end{array}$} & May & 72 & 52 & 62 & 2.1 & 0 & 64 & 47 & 56 & 3.5 \\
\hline & June & 79 & 57 & 68 & 2.4 & 0 & 74 & 58 & 66 & 3.1 \\
\hline & July & 84 & 59 & 72 & 2.2 & 0 & 79 & 64 & 72 & 3.2 \\
\hline & August & 81 & 54 & 68 & 3.0 & 0 & 78 & 63 & 71 & 3.2 \\
\hline & September & 79 & 49 & 64 & 1.9 & 0 & 70 & 56 & 63 & 3.9 \\
\hline & October & 68 & 46 & 57 & 2.6 & 0 & 59 & 45 & 52 & 3.6 \\
\hline & November & 57 & 38 & 48 & 0.96 & 0 & 48 & 36 & 42 & 4.0 \\
\hline \multirow{7}{*}{$\begin{array}{l}\text { Germansville, } \\
\text { Pennsylvania }\end{array}$} & May & 72 & 54 & 63 & 2.5 & 0 & 73 & 50 & 62 & 3.4 \\
\hline & June & 79 & 59 & 69 & 6.3 & 0 & 81 & 60 & 71 & 5.1 \\
\hline & July & 83 & 65 & 74 & 13 & 0 & 86 & 64 & 75 & 5.5 \\
\hline & August & 79 & 60 & 70 & 5.5 & 0 & 84 & 62 & 73 & 4.1 \\
\hline & September & 77 & 58 & 68 & 3.9 & 0 & 77 & 55 & 66 & 5.2 \\
\hline & October & 69 & 50 & 60 & 4.8 & 0 & 64 & 44 & 54 & 4.7 \\
\hline & November & 65 & 44 & 55 & 0.010 & 0 & 54 & 34 & 44 & 3.1 \\
\hline
\end{tabular}

Note: Weather data were reported from the planting date to the harvest date. Historical weather data were reported from 1981-2010 at sites Johnston, Iowa and Guelph, Ontario; 1987-2016 at sites Sheridan, Indiana and York, Nebraska; 2002-2016 at site Germansville, Pennsylvania; and 2007-2016 at site Fisk, Missouri. Definitions: monthly mean of daily maximum temperatures (Max), monthly mean of daily minimum temperatures (Min), and mean of Max and Min (Mean). 
Figure S1: Western Blot Results for ZMM28 Protein Derived from DP202216 Maize and Conventional Maize

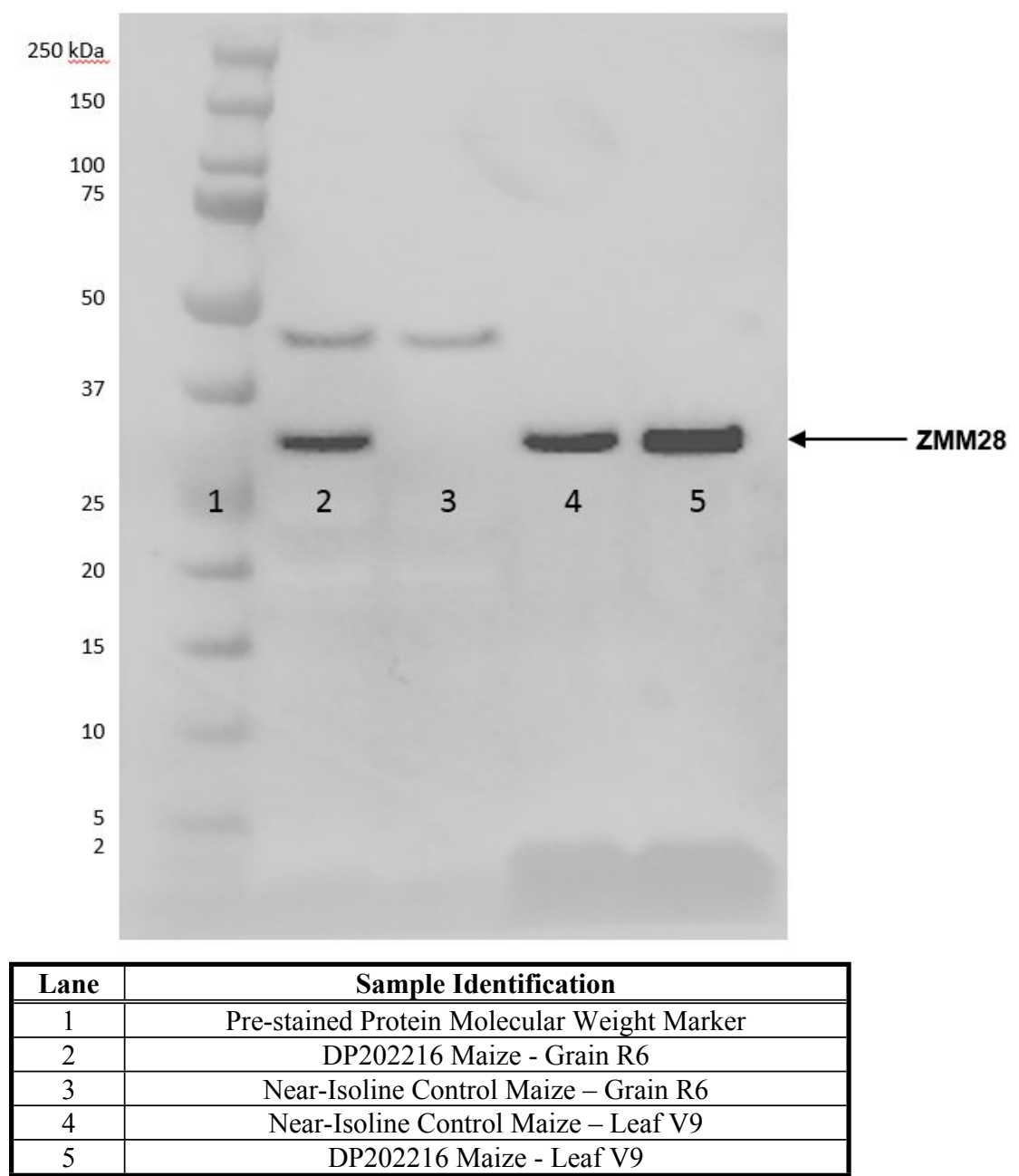

Note: kilodalton $(\mathrm{kDa})$. Molecular weight markers were included to provide a visual estimate that migration was within the expected range of the predicted molecular weight. A non-specific band ( $45 \mathrm{kDa})$ was detected by the antibody in the DP202216 maize and control maize grain.

Western blot analysis demonstrates that the ZMM28 protein in DP202216 maize (native and introduced) and control maize has the expected and equivalent size ( $28 \mathrm{kDa})$.

Western blot analysis conducted using ZMM28-specific antibodies does not differentiate between the native and introduced ZMM28 protein within DP202216 maize, so plant tissues from both DP202216 maize and conventional maize were selected, based on protein expression.

DP201226 maize expresses only the introduced ZMM28 protein in grain at the R6 growth stage (Lane 2), and both the native and introduced ZMM28 proteins in leaf at the V9 growth stage (Lane 5). The native and introduced ZMM28 proteins in the DP202216 grain and leaf samples migrated to the expected size $(28 \mathrm{kDa})$. The additional band migrating at $\sim 50 \mathrm{kDa}$ in DP202216 and conventional maize grain is a non-specific band. 
Conventional maize does not express the ZMM28 protein in grain at the R6 growth stage, and no ZMM28 band is detected on western blot (Lane 3). Conventional maize expresses the native ZMM28 protein in leaf at the V9 growth stage, and the native ZMM28 protein migrated to the expected size (28 kDa; Lane 4). The relative expression level is higher in the DP202216 leaf tissue. 
Figure S2: Western Blot Results for ZMM28 Protein Derived from DP202216 Maize and Conventional Maize

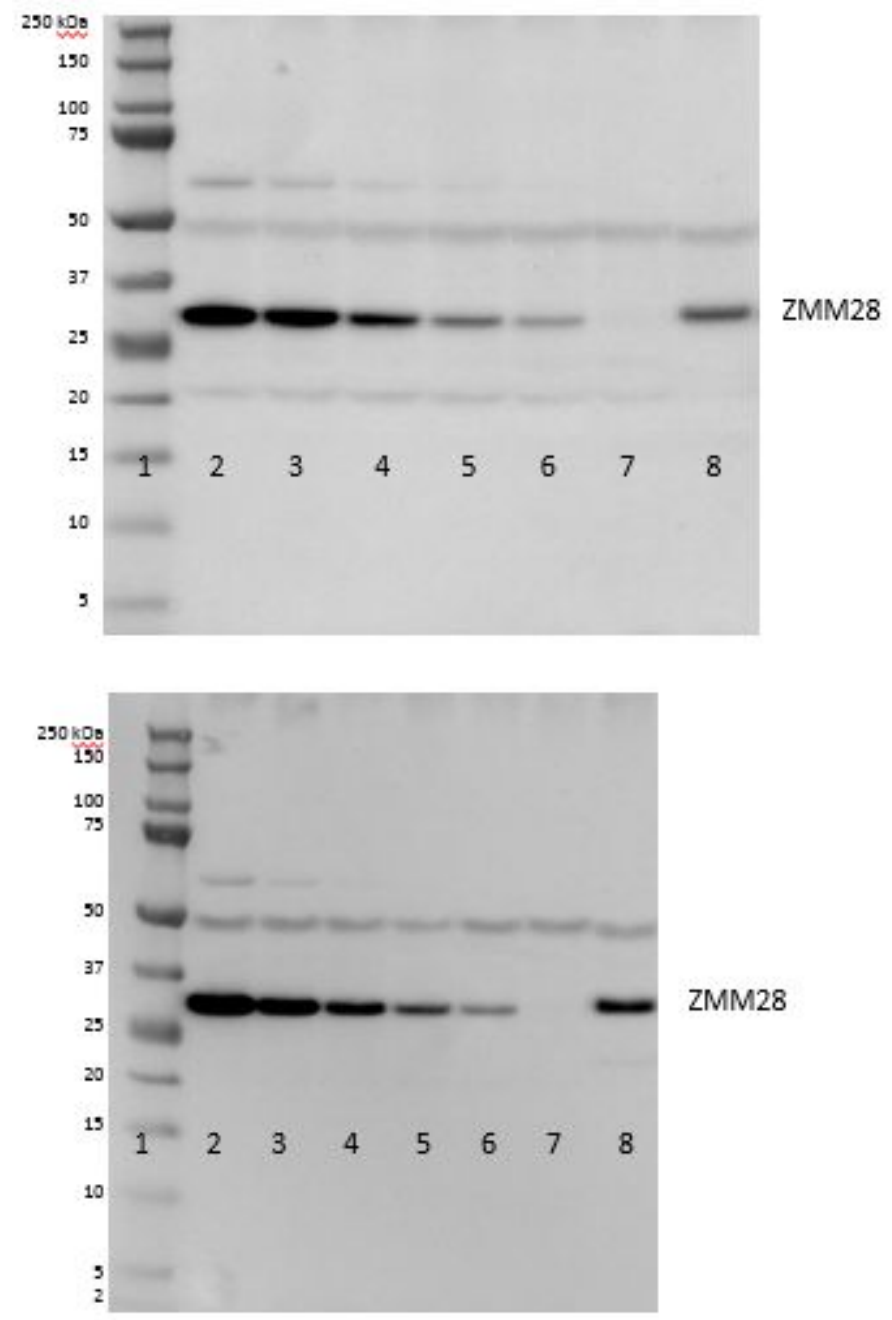

\begin{tabular}{|c|c|}
\hline Lane & Sample Identification \\
\hline \hline 1 & Pre-stained Protein Molecular Weight Marker \\
\hline 2 & ZMM28 Protein standard at $4.0 \mathrm{ng} / \mathrm{ml}$ \\
\hline 3 & ZMM28 Protein standard at $2.0 \mathrm{ng} / \mathrm{ml}$ \\
\hline 4 & ZMM28 Protein standard at $1.0 \mathrm{ng} / \mathrm{ml}$ \\
\hline 5 & ZMM28 Protein standard at $0.5 \mathrm{ng} / \mathrm{ml}$ \\
\hline 6 & ZMM28 Protein standard at $0.25 \mathrm{ng} / \mathrm{ml}$ \\
\hline 7 & ZMM28 Protein standard at $0 \mathrm{ng} / \mathrm{ml}$ \\
\hline 8 & DP202216 Maize - Grain $\mathrm{R} 6$ \\
\hline
\end{tabular}

Note: kilodalton $(\mathrm{kDa})$. Molecular weight markers were included to provide a visual estimate that migration was within the expected range of the predicted molecular weight.

Western blot analysis demonstrates that the ZMM28 protein in DP202216 maize grain (R6) and the characterized protein reference standard have the expected and equivalent size $(\sim 28 \mathrm{kDa})$. Characterization of the ZMM28 protein was achieved using sodium dodecyl sulfate polyacrylamide gel electrophoresis (SDS-PAGE) analysis, western blot analysis, amino acid composition analysis, mass determination of the intact protein by mass spectrometry, mass 
determination of tryptic and chymotryptic peptides by peptide mapping using matrix assisted laser desorption ionization mass spectrometry (MALDI-MS), and N-terminal amino acid sequencing.

MALDI-MS analysis of the microbially derived ZMM28 protein obtained a mass of 28,189.2 daltons $(\mathrm{Da})$, which is consistent with the expected mass of 28,187 $\mathrm{Da}$ of the protein sequence with truncation of three amino acids from the $\mathrm{C}$-terminus. 
Figure S3: Multiple sequence alignment of the zmm28 gene (cDNA sequence) in the B73 maize reference genome (Genbank accession no: NM_001111685.1; B73_reference), the native and introduced zmm28 genes from DP202216 maize (DP202216_Ntv and DP202216_Itd), and six varieties of sweet corn, including Country Gentleman (CG-A1), Early Golden Bantam (EGB_A1 and EGB_A2), Golden Jubilee (GJ_A1 and GJ_A2), Honey N' Pearl (HNP_A1 and HNP_A2), Incredible (I_A1), and Peaches and Cream (P\&C_A1). Three varieties have two alleles of the zmm28 gene sequence (EGB, GJ, and HNP) for a total of nine sweet corn cDNA sequences. The start codons and the stop codons of the coding sequences (CDS) are marked in the alignment with green and red, respectively.

B73 reference
202216 Ntv
202216 Itd
control
EGB_A1
EGB_A2
GJ_A 2
HND_A2
CG_A 1
GJ_A1
HND_A1
I_A 1
P\&CAA1

B73 reference $202 \overline{2} 16$ Ntv $202216^{-}$Itd control EGB_A1

EGB A2

GJ_ $\bar{A} 2$

$\mathrm{HN} \overline{\mathrm{P}} \mathrm{A} 2$

CG_A 1

GJ-A1

HNP A1

I_A $\overline{1}$

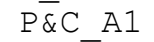

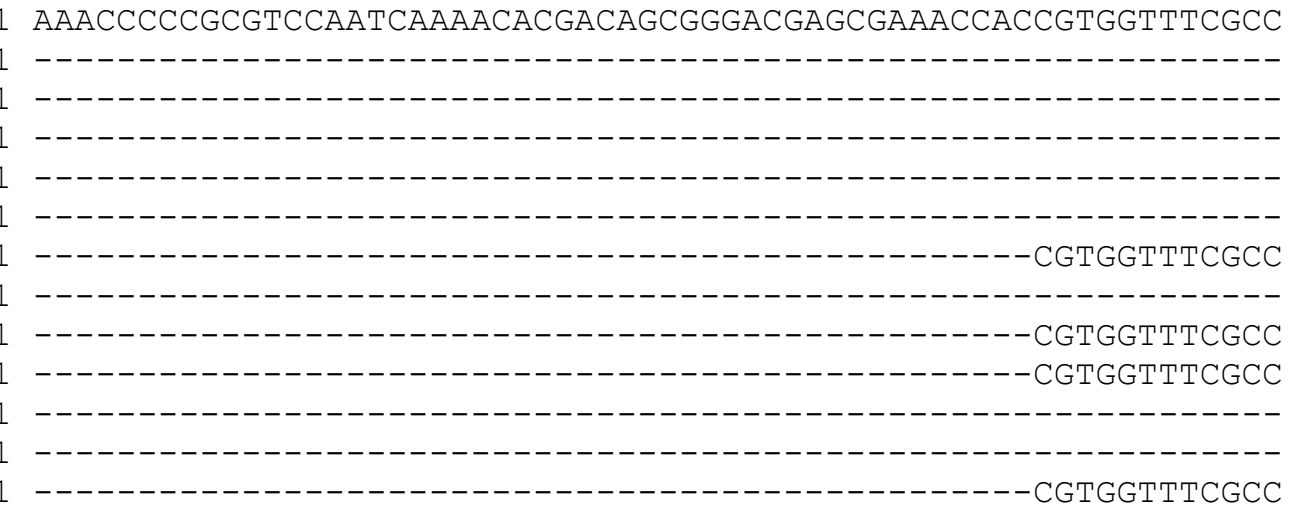


B73 reference 2022 16 Ntv $202216^{-}$Itd control EGB A1

EGB A2

GJ_A $\bar{A} 2$

$\mathrm{HN} \overline{\mathrm{P}} \mathrm{A} 2$

CG_ $\overline{\mathrm{A}} 1$

GJ A1

HNP A1

I_A $\overline{1}$

P\&D_A1

B73 reference 2022116_Ntv $202216^{-}$Itd control EGB A1

EGB A2

GJ_ $\overline{\mathrm{A}} 2$

$\mathrm{HN} \overline{\mathrm{P}} \mathrm{A} 2$

CG_A $\bar{A} 1$

GJ_A1

HNP A1

I_A $\overline{1}$

$\mathrm{P} \notin \mathrm{C} \_\mathrm{A} 1$
121

8 16

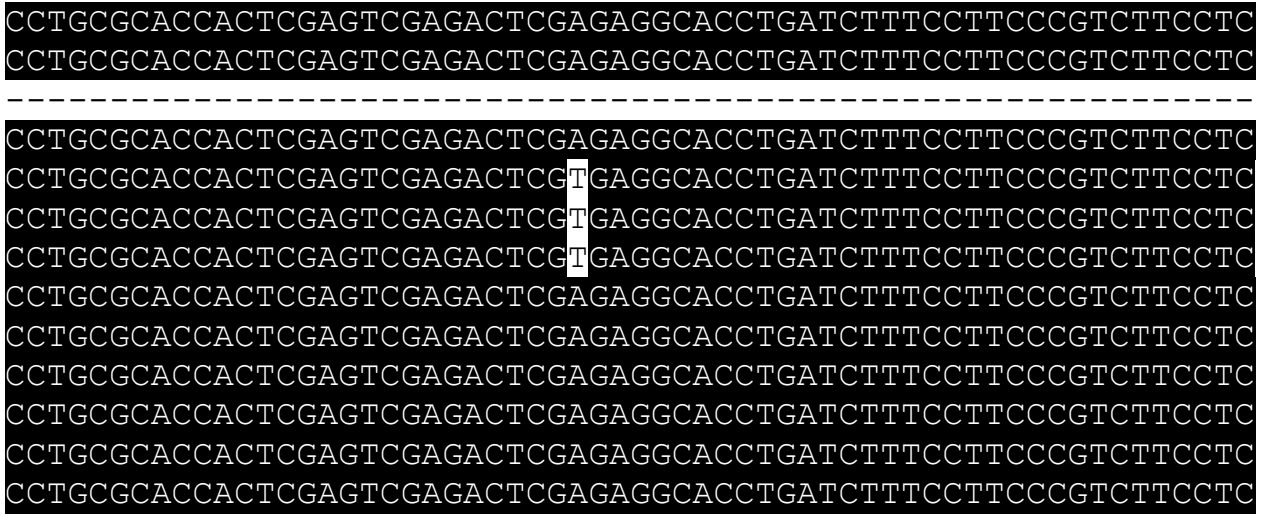

CCACACGTCCCCATCACCATTGCTGCGACGAGAGTGAGCGGGAGAGGGTAGGTGGCGAGC CACACGTCCCCATCACCATTGCTGCGACGAGAGTGAGCGGGAGAGGGTAGGTGGCGAG

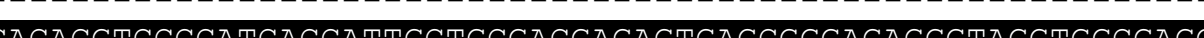

CCACACGTCCCCATCACCATTGCTGCGACGAGAGTGAGCGGGAGAGGGTAGGTGGCGAG CACACGTCCCCATCACCATTGCTGCGACGAGAGTGAGCGGGAGAGGGTAGGTGGCGAG CACACGTCCCCATCACCATTGCTGCGACGAGAGTGAGCGGGAGAGGGTAGGTGGCGAGG CACACGTCCCCATCACCATTGCTGCGACGAGAGTGAGCGGGAGAGGGTAGGTGGCGAG CACACGTCCCCATCACCATTGCTGCGACGAGAGTGAGCGGGAGAGGGTAGGTGGCGAG CACACGTCCCCATCACCATTGCTGCGACGAGAGTGAGCGGGAGAGGGTAGGTGGCGAGC CACACGTCCCCATCACCATTGCTGCGACGAGAGTGAGCGGGAGAGGGTAGGTGGCGAG CACACGTCCCCATCACCATTGCTGCGACGAGAGTGAGCGGGAGAGGGTAGGTGGCGAGC CACACGTCCCCATCACCATTGCTGCGACGAGAGTGAGCGGGAGAGGGTAGGTGGCGAGG
B73_reference $202216 \mathrm{NtV}$ $202216^{-}$Itd contrō EGB_A1

\section{1 \\ 128 \\ 1 \\ 136 \\ 146}

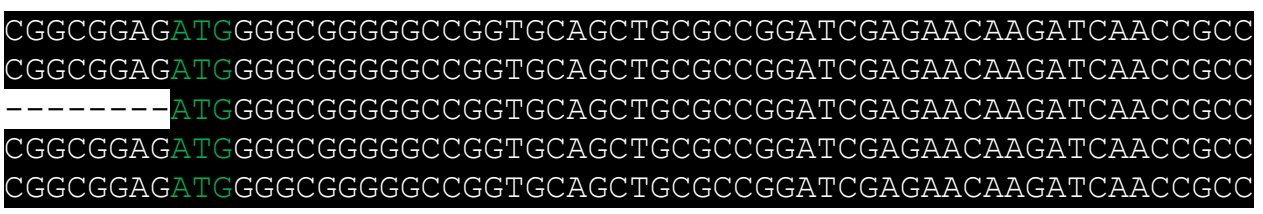




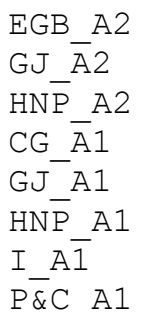

B73 reference $202 \overline{2} 16$ Ntv $202216^{-}$Itd control EGB A1

EGB $-A 2$

GJ_ $\bar{A} 2$

$\mathrm{HN} \overline{\mathrm{P}} \mathrm{A} 2$

CG_ $\bar{A} 1$

GJ-A1

$\mathrm{HN} \overline{\mathrm{P}} \mathrm{A} 1$

$I_{-} \mathrm{A} \overline{1}$

$\mathrm{P} \& \mathrm{C} \_\mathrm{A} 1$

B73 reference 2025̄16_Ntv $202216^{-}$Itd control EGB A1

EGB A2

GJ_ $\bar{A} 2$

$\mathrm{HN} \overline{\mathrm{P}} \mathrm{A} 2$

CG $\bar{A} 1$

GJ A1

$\mathrm{HN} \overline{\mathrm{P}} \mathrm{A} 1$

$I_{-} \mathrm{A} \overline{1}$

$\mathrm{P} \& \mathrm{C} \_\mathrm{A} 1$

B73_reference
146

193

145

193

193

146

146

193

301

188

53

196

206

206

253

205

253

253

206

206

253

361 248

113

256

266

266

313

265

313

313

266

266

313

421

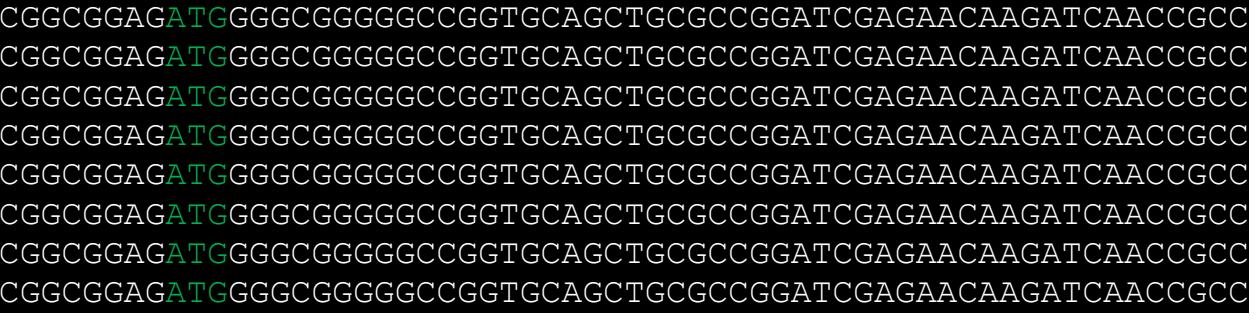

AGTGACCTTCTCCAAGCGCCGGAACGGGCTGCTGAAGAAGGCCCACGAGATCTCCGTGC AGGTGACCTTCTCCAAGCGCCGGAACGGGCTGCTGAAGAAGGCCCACGAGATCTCCGTGC GGTGACCTTCTCCAAGCGCCGGAACGGGCTGCTGAAGAAGGCCCACGAGATCTCCGTGC GGTGACCTTCTCCAAGCGCCGGAACGGGCTGCTGAAGAAGGCCCACGAGATCTCCGTG GGTGACCTTCTCCAAGCGCCGGAACGGGCTGCTGAAGAAGGCCCACGAGATCTCCGTGC GGTGACCTTCTCCAAGCGCCGGAACGGGCTGCTGAAGAAGGCCCACGAGATCTCCGTG GGTGACCTTCTCCAAGCGCCGGAACGGGCTGCTGAAGAAGGCCCACGAGATCTCCGTGC GGTGACCTTCTCCAAGCGCCGGAACGGGCTGCTGAAGAAGGCCCACGAGATCTCCGTGC GGTGACCTTCTCCAAGCGCCGGAACGGGCTGCTGAAGAAGGCCCACGAGATCTCCGTG GGTGACCTTCTCCAAGCGCCGGAACGGGCTGCTGAAGAAGGCCCACGAGATCTCCGTGC GGTGACCTTCTCCAAGCGCCGGAACGGGCTGCTGAAGAAGGCCCACGAGATCTCCGTGC GGTGACCTTCTCCAAGCGCCGGAACGGGCTGCTGAAGAAGGCCCACGAGATCTCCGTGC

TCTGCGACGCAGAGGTCGCGCTCATCGTCTTCTCCACTAAGGGGAAGCTCTACGAGTACT

CTGCGACGCAGAGGTCGCGCTCATCGTCTTCTCCACTAAGGGGAAGCTCTACGAGTACT CTGCGACGCAGAGGTCGCGCTCATCGTCTTCTCCACTAAGGGGAAGCTCTACGAGTAC: CTGCGACGCAGAGGTCGCGCTCATCGTCTTCTCCACTAAGGGGAAGCTCTACGAGTAC CTGCGACGCAGAGGTCGCGCTCATCGTCTTCTCCACTAAGGGGAAGCTCTACGAGTACT CTGCGACGCAGAGGTCGCGCTCATCGTCTTCTCCACTAAGGGGAAGCTCTACGAGTAC CTGCGACGCAGAGGTCGCGCTCATCGTCTTCTCCACTAAGGGGAAGCTCTACGAGTAC CTGCGACGCAGAGGTCGCGCTCATCGTCTTCTCCACTAAGGGGAAGCTCTACGAGTACT CTGCGACGCAGAGGTCGCGCTCATCGTCTTCTCCACTAAGGGGAAGCTCTACGAGTAC CTGCGACGCAGAGGTCGCGCTCATCGTCTTCTCCACTAAGGGGAAGCTCTACGAGTAC CTGCGACGCAGAGGTCGCGCTCATCGTCTTCTCCACTAAGGGGAAGCTCTACGAGTAC CTGCGACGCAGAGGTCGCGCTCATCGTCTTCTCCACTAAGGGGAAGCTCTACGAGTACT

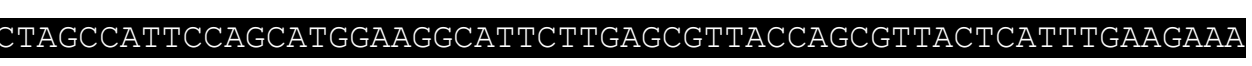


202216 Ntv $202216^{-}$Itd control

EGB_A1

$\mathrm{EGB}$ A2

GJ $\bar{A} 2$

$\mathrm{HN} \overline{\mathrm{P}} \mathrm{A} 2$

CG $\bar{A} 1$

GJ A1

$\mathrm{HN} \overline{\mathrm{P}} \mathrm{A} 1$

I $\mathrm{A} \overline{1}$

$\mathrm{P} \overline{\mathrm{C}} \mathrm{A} \mathrm{1}$

B73 reference

$202 \overline{2} 16 \mathrm{NtV}$

$202216^{-}$Itd

contrō

EGB_A1

$\mathrm{EGB}{ }^{-} \mathrm{A} 2$

GJ A2

HN $\bar{P}$ A2

CG_ $\bar{A} 1$

GJ A1

$\mathrm{HN} \overline{\mathrm{P}} \mathrm{A} 1$

I $A \overline{1}$

$\mathrm{P} \overline{\mathrm{C}} \mathrm{A}$ A 1

B73 reference $202 \overline{2} 16 \mathrm{Ntv}$

$202216^{\text {It }}$ It control

EGB A 1

EGB ${ }^{-}$A2

GJ A2

$\mathrm{HN} \overline{\mathrm{P}} \mathrm{A} 2$

CG $\bar{A} 1$

$\mathrm{GJ}^{-} \mathrm{A} 1$

HN $\bar{P}$ A 1

I_A $\overline{1}$
308

173

316

326

326

373

325

373

373

373
326

373

481

368

233

376

386

386

433

385

433

433

386

386

433

541
428

293

436

446

446

493

445

493

493

446

446
CTAGCCATTCCAGCATGGAAGGCATTCTTGAGCGTTACCAGCGTTACTCATTTGAAGAAA

TAGCCATTCCAGCATGGAAGGCATTCTTGAGCGTTACCAGCGTTACTCATTTGAAGAA

TAGCCATTCCAGCATGGAAGGCATTCTTGAGCGTTACCAGCGTTACTCATTTGAAGAA

TAGCCATTCCAGCATGGAAGGCATTCTTGAGCGTTACCAGCGTTACTCATTTGAAGAA

TAGCCATTCCAGCATGGAAGGCATTCTTGAGCGTTACCAGCGTTACTCATTTGAAGAA

TAGCCATTCCAGCATGGAAGGCATTCTTGAGCGTTACCAGCGTTACTCATTTGAAGAA

TAGCCATTCCAGCATGGAAGGCATTCTTGAGCGTTACCAGCGTTACTCATTTGAAGAA

TAGCCATTCCAGCATGGAAGGCATTCTTGAGCGTTACCAGCGTTACTCATTTGAAGAAA

TAGCCATTCCAGCATGGAAGGCATTCTTGAGCGTTACCAGCGTTACTCATTTGAAGAA

TAGCCATTCCAGCATGGAAGGCATTCTTGAGCGTTACCAGCGTTACTCATTTGAAGAA

TAGCCATTCCAGCATGGAAGGCATTCTTGAGCGTTACCAGCGTTACTCATTTGAAGAA

TAGCCATTCCAGCATGGAAGGCATTCTTGAGCGTTACCAGCGTTACTCATTTGAAGAAZ

\section{GGGCAGTACTTAACCCAAGTATTGAAGACCAGGCAAATTGGGGAGATGAATATGTCCGG}

GGCAGTACTTAACCCAAGTATTGAAGACCAGGCAAATTGGGGAGATGAATATGTCCGG

GGCAGTACTTAACCCAAGTATTGAAGACCAGGCAAATTGGGGAGATGAATATGTCCGGT

GGCAGTACTTAACCCAAGTATTGAAGACCAGGCAAATTGGGGAGATGAATATGTCCGGT

GGCAGTACTTAACCCAAGTATTGAAGACCAGGCAAATTGGGGAGATGAATATGTCCGG

GGCAGTACTTAACCCAAGTATTGAAGACCAGGCAAATTGGGGAGATGAATATGTCCGGT

GGCAGTACTTAACCCAAGTATTGAAGACCAGGCAAATTGGGGAGATGAATATGTCCGGT

GGCAGTACTTAACCCAAGTATTGAAGACCAGGCAAATTGGGGAGATGAATATGTCCGGT

GGCAGTACTTAACCCAAGTATTGAAGACCAGGCAAATTGGGGAGATGAATATGTCCGGT

GGCAGTACTTAACCCAAGTATTGAAGACCAGGCAAATTGGGGAGATGAATATGTCCGG

GGCAGTACTTAACCCAAGTATTGAAGACCAGGCAAATTGGGGAGATGAATATGTCCGGT

GGCAGTACTTAACCCAAGTATTGAAGACCAGGCAAATTGGGGAGATGAATATGTCCGGT

GGCAGTACTTAACCCAAGTATTGAAGACCAGGCAAATTGGGGAGATGAATATGTCCGGT

\section{TAAAATCCAAACTTGATGCACTTCAGAAGAGTCAAAGGCAGCTGTTAGGAGAACAATTGA}

AAAATCCAAACTTGATGCACTTCAGAAGAGTCAAAGGCAGCTGTTAGGAGAACAATTG

AAAATCCAAACTTGATGCACTTCAGAAGAGTCAAAGGCAGCTGTTAGGAGAACAATTGA

AAAATCCAAACTTGATGCACTTCAGAAGAGTCAAAGGCAGCTGTTAGGAGAACAATTG

AAAATCCAAACTTGATGCACTTCAGAAGAGTCAAAGGCAGCTGTTAGGAGAACAATTG

AAAATCCAAACTTGATGCACTTCAGAAGAGTCAAAGGCAGCTGTTAGGAGAACAATTGA

TAAAATCCAAACTTGATGCACTTCAGAAGAGTCAAAGGCAGCTGTTAGGAGAACAATTG

AAAATCCAAACTTGATGCACTTCAGAAGAGTCAAAGGCAGCTGTTAGGAGAACAATTGA

AAAATCCAAACTTGATGCACTTCAGAAGAGTCAAAGGCAGCTGTTAGGAGAACAATTG

TAAAATCCAAACTTGATGCACTTCAGAAGAGTCAAAGGCAGCTGTTAGGAGAACAATTG

TAAAATCCAAACTTGATGCACTTCAGAAGAGTCAAAGGCAGCTGTTAGGAGAACAATTGF

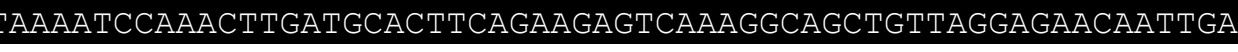


$P \& C \_A 1$

B73 reference 202216_Ntv

202216 Itd control

EGB_A1

EGB A2

GJ $\overline{\mathrm{A}} 2$

$\mathrm{HNP}$ A2

CG_ $\bar{A} 1$

GJ $A 1$

$\mathrm{HN} \overline{\mathrm{P}} \mathrm{A} 1$

I $\mathrm{A} \overline{1}$

$\overline{P \& C} A 1$

B73 reference 2022 16 Ntv

$202216^{-}$Itd

contrō

EGB_A1

EGB A2

GJ $\overline{\mathrm{A}} 2$

$\mathrm{HNP}$ A2

CG_ $\overline{\mathrm{A}} 1$

$\mathrm{GJ}^{-} \mathrm{A} 1$

HNP_A1

I $\mathrm{A} \overline{1}$

$\mathrm{P} \overline{\mathrm{C}} \mathrm{A} 1$

B73 reference $202 \overline{2} 16 \mathrm{Ntv}$

202216 Itd

contrō

EGB A1

EGB_A2

GJ $\overline{\mathrm{A}} 2$

HNP_A2
493

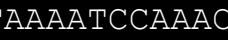

601

488

353

496

506

506

553

505

553

553

506

506

553

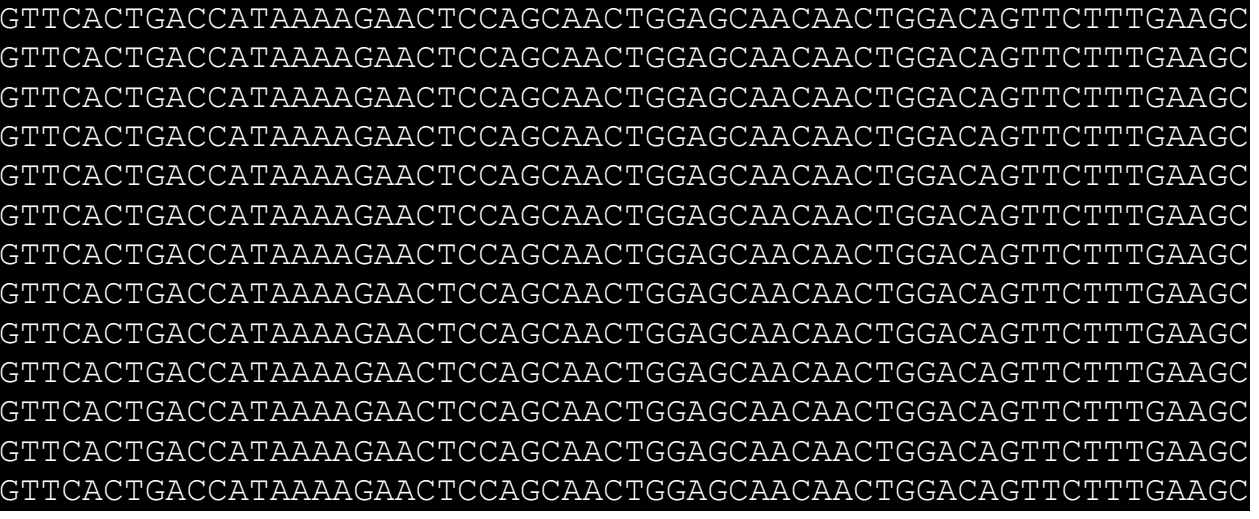

661 548

413

556

566

566

613

565

613

613

566

566

613

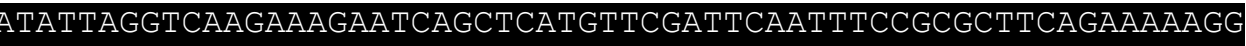

ATATTAGGTCAAGAAAGAATCAGCTCATGTTCGATTCAATTTCCGCGCTTCAGAAAAAG

ATATTAGGTCAAGAAAGAATCAGCTCATGTTCGATTCAATTTCCGCGCTTCAGAAAAAGC

TATTAGGTCAAGAAAGAATCAGCTCATGTTCGATTCAATTTCCGCGCTTCAGAAAAAGC

TATTAGGTCAAGAAAGAATCAGCTCATGTTCGATTCAATTTCCGCGCTTCAGAAAAAGC

ATATTAGGTCAAGAAAGAATCAGCTCATGTTCGATTCAATTTCCGCGCTTCAGAAAAAG

TATTAGGTCAAGAAAGAATCAGCTCATGTTCGATTCAATTTCCGCGCTTCAGAAAAAGC

TATTAGGTCAAGAAAGAATCAGCTCATGTTCGATTCAATTTCCGCGCTTCAGAAAAAG

TATTAGGTCAAGAAAGAATCAGCTCATGTTCGATTCAATTTCCGCGCTTCAGAAAAAGC

TATTAGGTCAAGAAAGAATCAGCTCATGTTCGATTCAATTTCCGCGCTTCAGAAAAAG

ATATTAGGTCAAGAAAGAATCAGCTCATGTTCGATTCAATTTCCGCGCTTCAGAAAAAG

TATTAGGTCAAGAAAGAATCAGCTCATGTTCGATTCAATTTCCGCGCTTCAGAAAAAGG

TATTAGGTCAAGAAAGAATCAGCTCATGTTCGATTCAATTTCCGCGCTTCAGAAAAAGC

721 AGAAAGCACTTACAGATCAAAACGGTGTCCTGCAAAAGTTCATGGAGGCAGAGAAGGAGA 608

473

616

626

626

673

625

AGAAAGCACTTACAGATCAAAACGGTGTCCTGCAAAAGTTCATGGAGGCAGAGAAGGAGA GAAAGCACTTACAGATCAAAACGGTGTCCTGCAAAAGTTCATGGAGGCAGAGAAGGAG

GAAAGCACTTACAGATCAAAACGGTGTCCTGCAAAAGTTCATGGAGGCAGAGAAGGAG

GAAAGCACTTACAGATCAAAACGGTGTCCTGCAAAAGTTCATGGAGGCAGAGAAGGAGZ

GAAAGCACTTACAGATCAAAACGGTGTCCTGCAAAAGTTCATGGAGGCAGAGAAGGAG

GAAAGCACTTACAGATCAAAACGGTGTCCTGCAAAAGTTCATGGAGGCAGAGAAGGAGI

GAAAGCACTTACAGATCAAAACGGTGTCCTGCAAAAGTTCATGGAGGCAGAGAAGGAGA 
CG_A1
GJ_A1
HND_A1
I_A $\overline{1}$
$\mathrm{P} \otimes \mathrm{C} A 1$

B73 reference $202 \overline{2} 16$ Ntv $202216^{-}$Itd control EGB_A1 EGB $A 2$

GJ A2

$\mathrm{HN} \overline{\mathrm{P}} \mathrm{A} 2$

CG $\bar{A} 1$

GJ-A1

$\mathrm{HN} \overline{\mathrm{P}} \mathrm{A} 1$

I_A $\overline{1}$

$\mathrm{P} \overline{\mathrm{C}} \_\mathrm{A} 1$

B73 reference $202 \overline{2} 16$ Ntv $202216^{-}$Itd contrō EGB_A1 $\mathrm{EGB}-\mathrm{A} 2$

GJ $\bar{A} 2$

$\mathrm{HN} \overline{\mathrm{P}} \mathrm{A} 2$

CG $\bar{A} 1$

$\mathrm{GJ}^{-} \mathrm{A} 1$

$\mathrm{HN} \overline{\mathrm{P}} \mathrm{A} 1$

$I_{A} \overline{\overline{1}}$

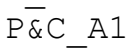

B73 reference $202 \overline{2} 16$ Ntv $202216^{-}$Itd control
673

673

626

626

673

781

668

533

676

686

686

733

685

733

733

686

686
733

733

841

728

593

736

746

746

793

745

793

793

746

746

793

901

788

653

796
AGAAAGCACTTACAGATCAAAACGGTGTCCTGCAAAAGTTCATGGAGGCAGAGAAGGAGA

GAAAGCACTTACAGATCAAAACGGTGTCCTGCAAAAGTTCATGGAGGCAGAGAAGGAGA GAAAGCACTTACAGATCAAAACGGTGTCCTGCAAAAGTTCATGGAGGCAGAGAAGGAG GAAAGCACTTACAGATCAAAACGGTGTCCTGCAAAAGTTCATGGAGGCAGAGAAGGAGA GAAAGCACTTACAGATCAAAACGGTGTCCTGCAAAAGTTCATGGAGGCAGAGAAGGAGZ

\section{AAACAAGGCTTTGATGAACGCGCAGCTCCGGGAGCAGCAAAATGGAGCATCAACAAGC}

AAACAAGGCTTTGATGAACGCGCAGCTCCGGGAGCAGCAAAATGGAGCATCAACAAGC

AAACAAGGCTTTGATGAACGCGCAGCTCCGGGAGCAGCAAAATGGAGCATCAACAAGC

AAACAAGGCTTTGATGAACGCGCAGCTCCGGGAGCAGCAAAATGGAGCATCAACAAGC'

AAACAAGGCTTTGATGAACGCGCAGCTCCGGGAGCAGCAAAATGGAGCATCAACAAGC

AAACAAGGCTTTAATGAACGCGCAGCTCCGGGAGCAGCAAAATGGAGCATCAACAAGC

AAACAAGGCTTTAATGAACGCGCAGCTCCGGGAGCAGCAAAATGGAGCATCAACAAGC

AAACAAGGCTTTGATGAACGCGCAGCTCCGGGAGCAGCAAAATGGAGCATCAACAAGC

AAAACAAGGCTTTGATGAACGCGCAGCTCCGGGAGCAGCAAAATGGAGCATCAACAAGC

AAACAAGGCTTTGATGAACGCGCAGCTCCGGGAGCAGCAAAATGGAGCATCAACAAGC

AAACAAGGCTTTGATGAACGCGCAGCTCCGGGAGCAGCAAAATGGAGCATCAACAAGC

AAACAAGGCTTTGATGAACGCGCAGCTCCGGGAGCAGCAAAATGGAGCATCAACAAGC

AAACAAGGCTTTGATGAACGCGCAGCTCCGGGAGCAGCAAAATGGAGCATCAACAAGC

CCCCATCACTTTCACCACCAATAGTTCCAGATTCCATGCCAACTCTAAATATAGGGCCAT

CCCATCACTTTCACCACCAATAGTTCCAGATTCCATGCCAACTCTAAATATAGGGCCA

CCCATCACTTTCACCACCAATAGTTCCAGATTCCATGCCAACTCTAAATATAGGGCCA

CCCATCACTTTCACCACCAATAGTTCCAGATTCCATGCCAACTCTAAATATAGGGCCA

CCCATCACTTTCACCACCAATAGTTCCAGATTCCATGCCAACTCTAAATATAGGGCCA

CCCATCACTTTCACCACCAATAGTTCCAGATTCCATGCCAACTCTAAATATAGGGCCA

CCCATCACTTTCACCACCAATAGTTCCAGATTCCATGCCAACTCTAAATATAGGGCCA'

CCCATCACTTTCACCACCAATAGTTCCAGATTCCATGCCAACTCTAAATATAGGGCCAT

CCCATCACTTTCACCACCAATAGTTCCAGATTCCATGCCAACTCTAAATATAGGGCCAT

CCCATCACTTTCACCACCAATAGTTCCAGATTCCATGCCAACTCTAAATATAGGGCCAT

CCCATCACTTTCACCACCAATAGTTCCAGATTCCATGCCAACTCTAAATATAGGGCCAT

CCCATCACTTTCACCACCAATAGTTCCAGATTCCATGCCAACTCTAAATATAGGGCCA

CCCATCACTTTCACCACCAATAGTTCCAGATTCCATGCCAACTCTAAATATAGGGCCAT

GTCAACATAGAGGGGCAGCAGAATCTGAGTCTGAACCGTCTCCTGCTCCTGCACAAGCAA

TCAACATAGAGGGGCAGCAGAATCTGAGTCTGAACCGTCTCCTGCTCCTGCACAAGCA

GTCAACATAGAGGGGCAGCAGAATCTGAGTCTGAACCGTCTCCTGCTCCTGCACAAGCAA

GTCAACATAGAGGGGCAGCAGAATCTGAGTCTGAACCGTCTCCTGCTCCTGCACAAGCAA 


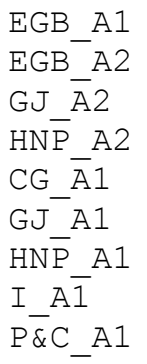

B73 reference $202 \overline{2} 16 \mathrm{Ntv}$

$202216^{-}$Itd contrō

EGB A1

EGB ${ }^{-}$A2

GJ $\bar{A} 2$

$\mathrm{HN} \overline{\mathrm{P}} \_\mathrm{A} 2$

CG $\bar{A} 1$

GJ A1

$\mathrm{HN} \overline{\mathrm{P}} \mathrm{A} 1$

I $A \overline{1}$

$\mathrm{P} \bar{\S} \mathrm{C} \_\mathrm{A} 1$

B73 reference 1021 $202 \overline{2} 16 \mathrm{Ntv}$

$202216^{-}$Itd contrō

EGB A1

EGB A2

GJ $\bar{A} 2$

$\mathrm{HN} \overline{\mathrm{P}} \mathrm{A} 2$

CG $\bar{A} 1$

GJ $\mathrm{A} 1$

$\mathrm{HN} \overline{\mathrm{P}} \mathrm{A} 1$

I $\bar{A} \overline{1}$

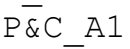

866

913

866

913

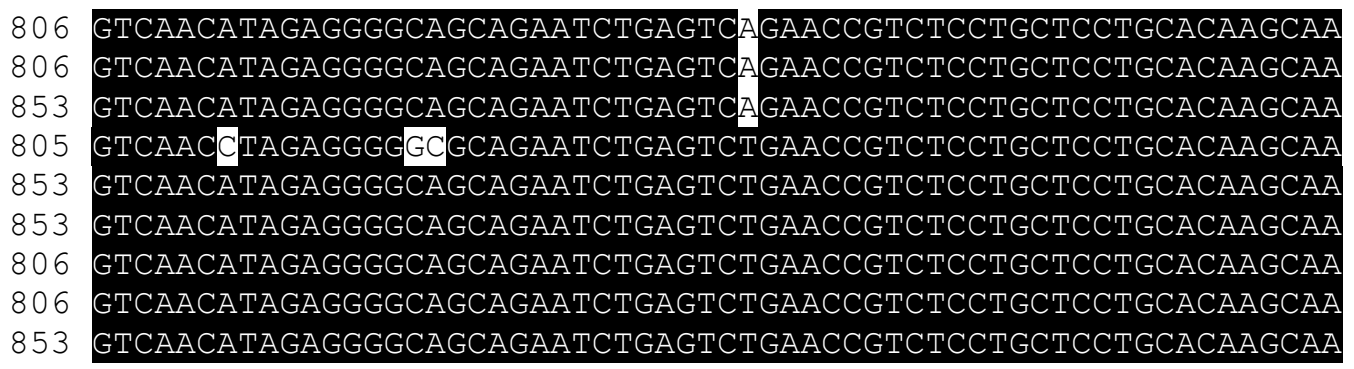

961

848

713

856

866

913

865

913

866

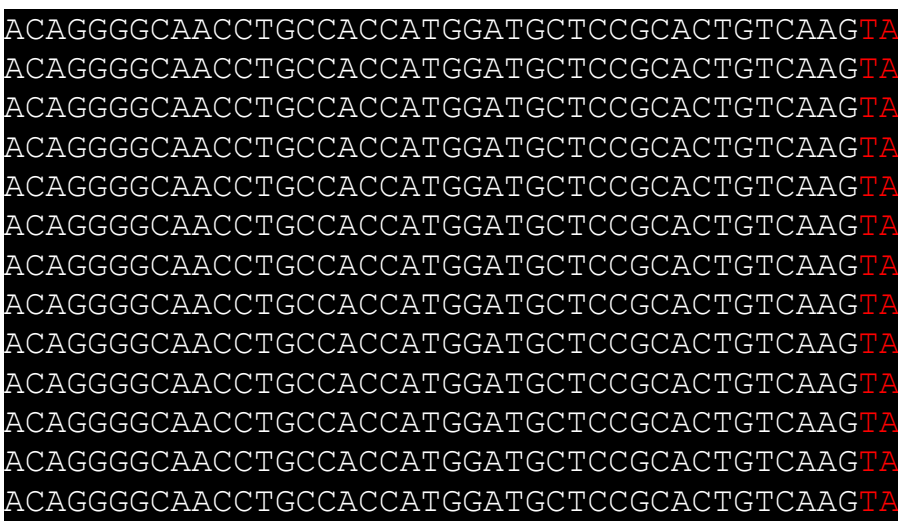

ACAGGTGAGGTCTTCCC

-

CAGGTGAGGTCTTCCC

ACAGGTGAGGTCTTCCC

ACAGGTGAGGTCTTCCC

ACAGGTGAGGTCTTCCC

CAGGTGAGGTCTTCC

ACAGGTGAGGTCTTCCC

CAGGTGAGGTCTTCC

ACAGGTGAGGTCTTCCC

ACAGGTGAGGTCTTCC

CAGGTGAGGTCTTCCC
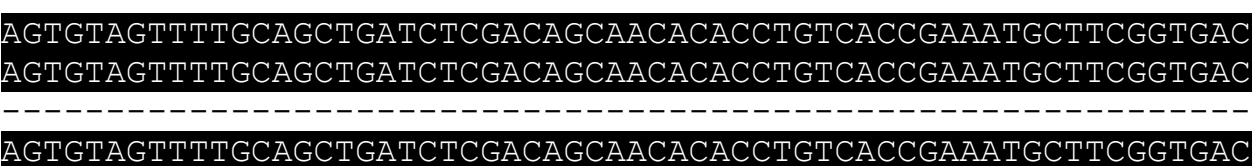

AGTGTAGTTTTGCAGCTGATCTCGACAGCAACACACCTGTCACCGAAATGCTTCGGTGAC

AGTGTAGTTTTGCAGCTGATCTCGACAGCAACACACCTGTCACCGAAATGCTTCGGTGAC

AGTGTAGTTTTGCAGCTGATCTCGACAGCAACACACCTGTCACCGAAATGCTTCGGTGAC

AGTGTAGTTTTGCAGCTGATCTCGACAGCAACACACCTGTCACCGAAATGCTTCGGTGA

AGTGTAGTTTTGCAGCTGATCTCGACAGCAACACACCTGTCACCGAAATGCTTCGGTGAC

AGTGTAGTTTTGCAGCTGATCTCGACAGCAACACACCTGTCACCGAAATGCTTCGGTGA

AGTGTAGTTTTGCAGCTGATCTCGACAGCAACACACCTGTCACCGAAATGCTTCGGTGAC

AGTGTAGTTTTGCAGCTGATCTCGACAGCAACACACCTGTCACCGAAATGCTTCGGTGAC

973 AGTGTAGTTTTGCAGCTGATCTCGACAGCAACACACCTGTCACCGAAATGCTTCGGTGAC 
B73 reference 1081 $202 \overline{2} 16$ Ntv $202216^{-}$Itd control EGB A1

EGB A2

GJ_ $\bar{A} 2$

HNP_A2

CG_A 1

$\mathrm{GJ}^{-} \mathrm{A} 1$

HNP A1

$I_{-} \mathrm{A} \overline{1}$

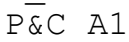

B73 reference 1141

2025̄16_Ntv 1028 $202216^{-}$Itd control EGB_A1

EGB $A 2$

GJ_ $\bar{A} 2$

$\mathrm{HN} \overline{\mathrm{P}} \mathrm{A} 2$

CG_ $\bar{A} 1$

GJ'A1

$\mathrm{HN} \overline{\mathrm{P}} \mathrm{A} 1$

I_A $\overline{1}$

$\mathrm{P} \overline{\overline{ }} \mathrm{C} \quad \mathrm{A} 1$

B73 reference 1201

$202 \overline{2} 16$ Ntv 1088 $202216^{-}$Itd control EGB A1

EGB A2

GJ_ $\bar{A} 2$

$\mathrm{HN} \overline{\mathrm{P}} \mathrm{A} 2$

CG $\bar{A} 1$

GJ A1

HNP_A 1
968

976

986

986

1033

985

1033

1033

986

986

1033

1036

1046

1046

1093

1045

1093

1093

1046

1046

1093

1096

1106

1106

1153

1105

1153

1153

1106

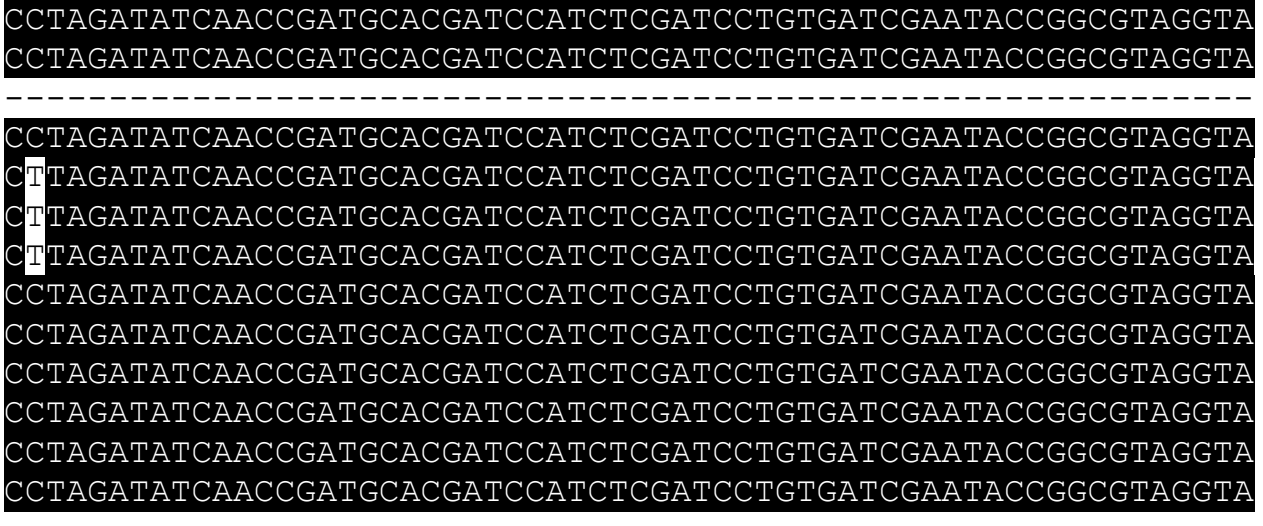

CTACCAATAAAGTCAGGCCCCGTCAAATGCTTTACATGGTGATTGAAGTCTGGAGCTTC TACCAATAAAGTCAGGCCCCGTCAAATGCTTTACATGGTGATTGAAGTCTGGAGCTTCT

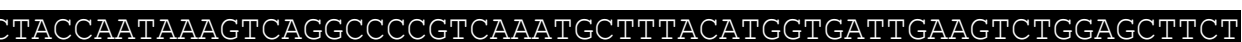

CTACTAATAAAGTCAGGCCCCGTCAAATGCTTTACATGGTGATTGAAGTCTGGAGCTTC

CTACTAATAAAGTCAGGCCCCGTCAAATGCTTTACATGGTGATTGAAGTCTGGAGCTTC

TACTAATAAAGTCAGGCCCCGTCAAATGCTTTACATGGTGATTGAAGTCTGGAGCTTCT

TACTAATAAAGTCAGGCCCCGTCAAATGCTTTACATGGTGATTGAAGTCTGGAGCTTCI TACCAATAAAGTCAGGCCCCGTCAAATGCTTTACATGGTGATTGAAGTCTGGAGCTTC TACCAATAAAGTCAGGCCCCGTCAAATGCTTTACATGGTGATTGAAGTCTGGAGCTTC TACCAATAAAGTCAGGCCCCGTCAAATGCTTTACATGGTGATTGAAGTCTGGAGCTTCI TACCAATAAAGTCAGGCCCCGTCAAATGCTTTACATGGTGATTGAAGTCTGGAGCTTCI CTACCAATAAAGTCAGGCCCCGTCAAATGCTTTACATGGTGATTGAAGTCTGGAGCTTCT

CAGCACCACCATATATTGTGTTCAGTTCCTGTGTTTATTGCCATAGCTACCCTTTTTTT-

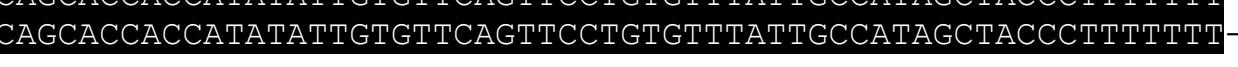

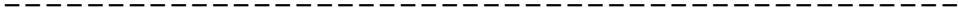

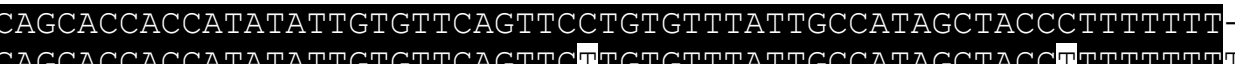

CAGCACCACCATATATTGTGTTCAGTTCTTGTGTTTATTGCCATAGCTACCTTTTTTTTT

CAGCACCACCATATATTGTGTTCAGTTC

GTGTTTATTGCCATAGCTAC

GTGTTTATTGCCATAGCTACCITTTTTTT

CAGCACCACCATATATTGTGTTCAGTTCTTGTGTTTATTGCCATAGCTACCTTTTTTTT-

CAGCACCACCATATATTGTGTTCAGTTCCTGTGTTTATTGCCATAGCTACCCTTTTTTT-

CAGCACCACCATATATTGTGTTCAGTTCCTGTGTTTATTGCCATAGCTACCCTTTTTTT-

CAGCACCACCATATATTGTGTTCAGTTCCTGTGTTTATTGCCATAGCTACCCTTTTTTT 


\begin{tabular}{|c|c|c|}
\hline _A1 & & \\
\hline $\bar{\varepsilon} \mathrm{C} \_\mathrm{A} 1$ & & GTTCAGTTCCTGTGTTTATTGCCATAGCTACCCTTTTTT: \\
\hline 73 reference & 1260 & \\
\hline $2 \overline{2} 16 \_N t v$ & 1147 & 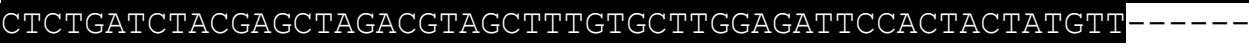 \\
\hline 2216 Itd & & \\
\hline ontrō̄ & 1155 & CTGATCTACGAGCTAGACGTAGCTTTGTGCTTGGAGATTCCACTACTATGTT- \\
\hline GB A1 & 1166 & СTCTGATCTACGAGCTAGACGTAGCTTTGTGCTTGGAGATTCCACTACTATGTTTCCT \\
\hline $\mathrm{BB}-\mathrm{A} 2$ & 1166 & СTCTGATCTACGAGCTAGACGTAGCTTTGTGCTTGGAGATTCCACTACTATGTTTCCI \\
\hline J_ & 1213 & СTCTGATCTACGAGCTAGACGTAGCTTTGTGCTTGGAGATTCCACTACTATGTTTCCI \\
\hline $\mathrm{N} \overline{\mathrm{P}} \mathrm{A} 2$ & 1164 & СTCTGATCTACGAGCTAGACGTAGCTTTGTGCTTGGAGATTCCACTACTATGTTTCO \\
\hline$G_{-} \bar{A} 1$ & 1212 & СTCTGATCTACGAGCTAGACGTAGCTTTGTGCTTGGAGATTCCACTACTATGTTTCCT \\
\hline & 1212 & СTCTGATCTACGAGCTAGACGTAGCTTTGTGCTTGGAGATTCCACTACTATGTTTCCT \\
\hline $\mathrm{N} \overline{\mathrm{P}} \mathrm{A} 1$ & 1165 & СTCTGATCTACGAGCTAGACGTAGCTTTGTGCTTGGAGATTCCACTACTATGTTTCC \\
\hline I_A $\mathrm{i}$ & 1165 & СTCTGATCTACGAGCTAGACGTAGCTTTGTGCTTGGAGATTCCACTACTATGTTTC \\
\hline & & TGATCTACGAGCTAGACGTAGCTTTGTGCTTGGAGATTCCACTACTATGTTTCC \\
\hline
\end{tabular}
$202 \overline{2} 16 \mathrm{Ntv}$ 202216 Itd control

EGB_A1

$\mathrm{EGB}$ A2

GJ $\bar{A} 2$

HND $\bar{P}$ A2

CG_ $\overline{\mathrm{A}} 1$

GJ_A1

$\operatorname{HN} \overline{\mathrm{P}} \mathrm{A} 1$

I $A \overline{1}$

$\mathrm{P} \bar{\AA} \mathrm{C} \_\mathrm{A} 1$

1226
1226
1273
1224
1272
1272
1225
1225
1272

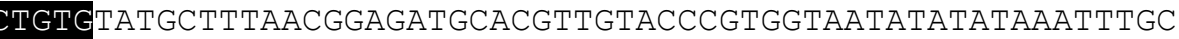

泟

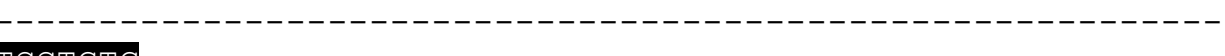

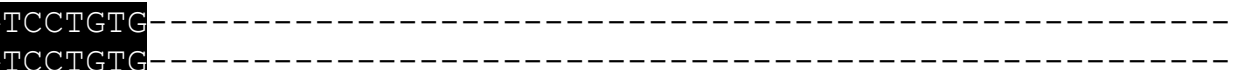

GTCCTGTGTATGCTTTAACGGAGATGCACGTTGTACCCGTGGTAATATATATAAATTTGC

GTCCTGTG

GTCCTGTGTATGCTTTAACGGAGATGCACGTTGTACCCGTGGTAATATATATAAATTTGC

GTCCTGTGTATGCTTTAACGGAGATGCACGTTGTACCCGTGGTAATATATATAAATTTGC

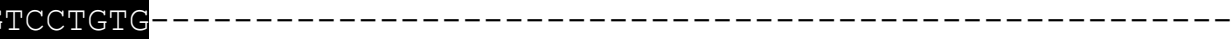

TCCTGTC-

GTCTGTGTATGCTTTAACGGAGATGCACGTTGTACCCGTGGTAATATATATAAATTTGC

B73 reference 1380 GTTGTAAAGATCAAGTGGCTCTTTTCTCCGAAATGTTATGAAGTCTCAGGTGTCAAACAT 202216 Ntv $202216^{-}$Itd control EGB A1 $\mathrm{EGB}{ }^{-} \mathrm{A} 2$ GJ_A $\bar{A} 2$ -----------------------------------------------------------

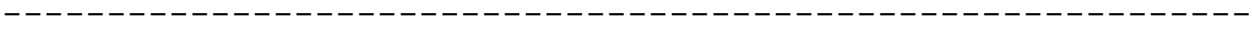
-------------------------------------------------------- 


\section{Supporting Information}

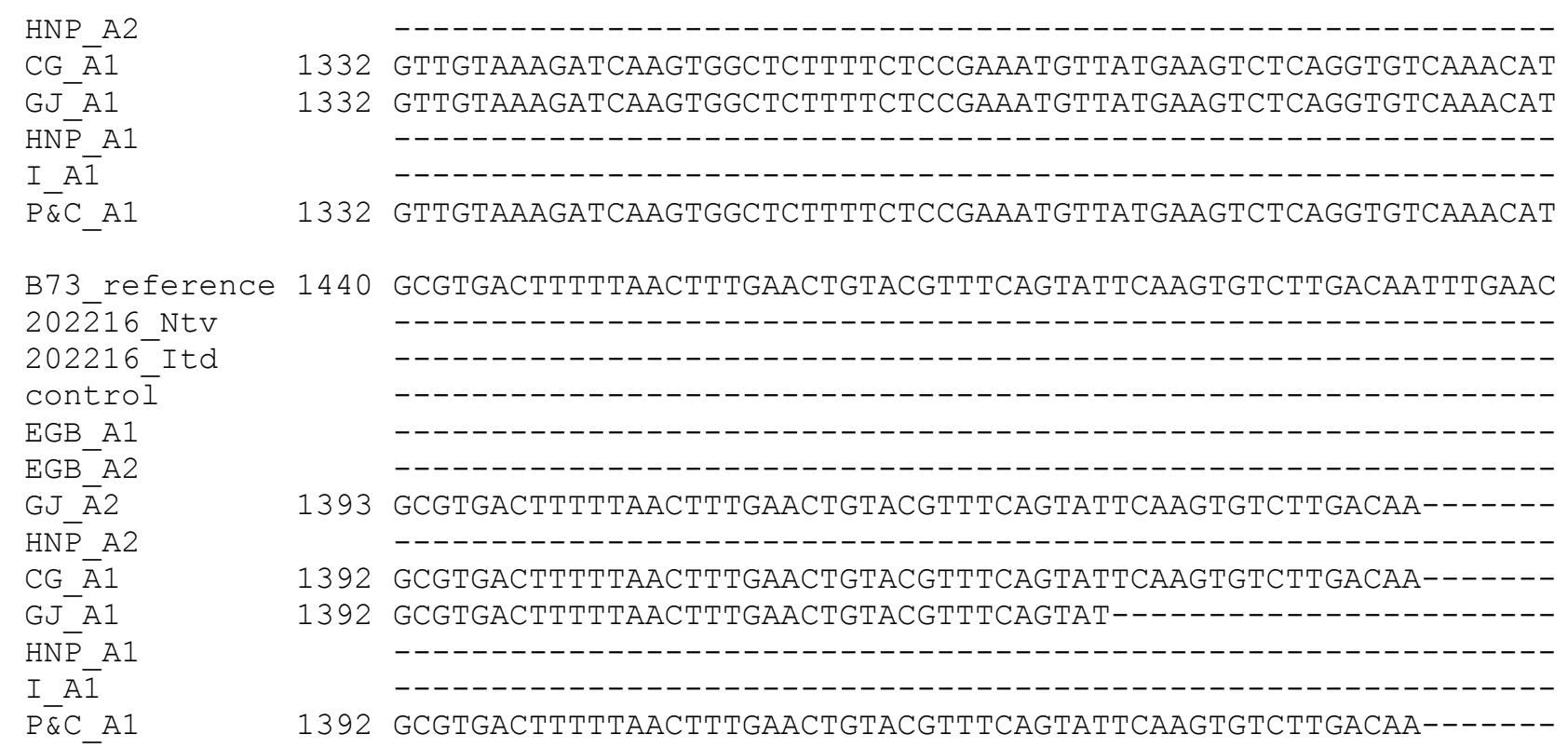

B73 reference 1500 CGCGT

$202 \overline{2} 16 \mathrm{NtV}$

$202216^{-}$Itd

control -.---

EGB A1 ----

$\mathrm{EGB}$ A2 ----

GJ $\overline{\mathrm{A}} 2$

$\mathrm{HN} \overline{\mathrm{P}} \mathrm{A} 2$

$\mathrm{CG} \overline{\mathrm{A}} 1$

GJ A1 -----

$\mathrm{HN} \overline{\mathrm{P}} \_\mathrm{A} 1$

I $\mathrm{A} \overline{1}$

$\mathrm{P} \bar{\AA} \mathrm{C} \_\mathrm{A} 1$ 